\title{
A Survey of the Perceived Text Adaptation Needs of Adults with Autism
}

\author{
Victoria Yaneva, Constantin Orăsan, Le An Ha and Natalia Ponomareva \\ Research Institute in Information and Language Processing, \\ University of Wolverhampton, UK \\ \{v.yaneva, c.orasan, ha.l.a, n.ponomareva\}@wlv.ac.uk
}

\begin{abstract}
NLP approaches to automatic text adaptation often rely on user-need guidelines which are generic and do not account for the differences between various types of target groups. One such group are adults with high-functioning autism, who are usually able to read long sentences and comprehend difficult words but whose comprehension may be impeded by other linguistic constructions. This is especially challenging for real-world usergenerated texts such as product reviews, which cannot be controlled editorially and are thus in a stronger need of automatic adaptation. To address this problem, we present a mixedmethods survey conducted with 24 adult webusers diagnosed with autism and an agematched control group of 33 neurotypical participants. The aim of the survey is to identify whether the group with autism experiences any barriers when reading online reviews, what these potential barriers are, and what NLP methods would be best suited to improve the accessibility of online reviews for people with autism. The group with autism consistently reported significantly greater difficulties with understanding online product reviews compared to the control group and identified issues related to text length, poor topic organisation, identifying the intention of the author, trustworthiness, and the use of irony, sarcasm and exaggeration.
\end{abstract}

\section{Introduction}

The aim of automatic text adaptation (also known as automatic text simplification) is to make the meaning of texts more accessible to specific target groups such as language learners or people with cognitive disabilities. To achieve this, the development of automatic systems is driven by guidelines that describe the needs of the target group, but very often these guidelines are generic. For example, one of the most authoritative sources of such guidelines for people with cognitive disabilities, the European Guidelines for the Production of Easy-to-Read Information (Freyhoff et al., 1998), lists requirements that fit the profile of people with moderate to severe comprehension deficits, but not those of more highly able individuals. As a result, the majority of text simplification strategies aim to reduce sentence and word complexity, while approaches to other aspects of text adaptation (e.g. clarifying the opinion of the author or strengthening the text organization) receive less attention.

In this paper we address this issue by providing insight into the needs of a specific subgroup, people with high-functioning autism, who are known to be able to read and comprehend complex texts, but may struggle with specific aspects of their comprehension. For example, readers with highfunctioning autism are usually able to cope with the meaning of complex words but are known to struggle with non-literal language or with combining the meaning of individual text components into a meaningful whole (see Section 2).

These difficulties are particularly challenging when interacting with texts that were not controlled editorially and may create barriers for people with autism to interact with the web, make informed decisions, and being an active part of the economy. One such type of text is the user feedback on goods and services, which is increasingly used to guide decision making in many aspects of life, from travel, entertainment, and shopping to education, social care, and policy making (Eynon and Margetts, 2007). This feedback usually comprises numerical ratings and written reviews submitted by users. A survey of online shoppers in the UK showed that product reviews have the greatest influence on purchasing decisions, greater than that of expert reviews or advice from friends (Fretwell et al., 2013). Unlike other types of Web content, whose accessibility can be controlled edi- 
torially, product reviews in user feedback are produced spontaneously by a large, dynamic, and heterogeneous population of writers. Unsurprisingly, this feedback, which represents a large proportion of content available on the Web, is of varying levels of accessibility.

The paper presents a mixed-methods survey conducted with 24 adult web-users diagnosed with autism and an age-matched control group of 33 neurotypical participants. The aim of the survey was to identify whether the group with autism: i) experienced any barriers when reading online reviews, ii) what these potential barriers were, and iii) what automatic methods would be best suited to improve the accessibility of online reviews for people with autism. To the best of our knowledge, this is the first time when the perception of online product reviews has been investigated in terms of its accessibility for people with autism.

The next section presents information on the reading difficulties of people with autism.

\section{Background}

\subsection{Autism Spectrum Disorder and Reading}

Autism Spectrum Disorder (ASD) is a developmental disorder with neural origin characterised by impairment in communication and social interaction (American Psychiatric Association, 2013) and is known to affect about 1 in 100 people in the UK (Brugha et al., 2011). Language comprehension difficulties in autism cover phenomena such as difficulties in syntax processing of long sentences (Whyte et al., 2014), resolving ambiguity in meaning (Happe, F., and Frith, U, 2006), and identifying pronoun referents ( $\mathrm{O}$ 'Connor and Klein, 2004), as well as having difficulties in figurative language comprehension and making pragmatic inferences (MacKay and Shaw, 2004). These difficulties, together with the specific cognitive profile of individuals with autism (e.g., differences in the Theory of Mind (Baron-Cohen, 2000)) may lead to secondary issues such as challenges with identifying author intent and subtler nuances of meaning. In addition, web users with autism have been consistently shown to have different information searching strategies when processing web pages (Eraslan et al., 2017; Yaneva et al., 2018; Eraslan et al., 2019; Yaneva et al., 2019), which relate to differences in visual attention. As a result of these difficulties, information contained in online user feedback can be less accessible for people with autism.

\subsection{Automatic Text Adaptation for Adults with Autism}

In terms of systems aimed at making text more accessible for autistic individuals who are fairly able, the OpenBook tool ${ }^{1}$ is the most comprehensive existing system to date. The tool provides semi-automatic conversion of text documents by reducing syntactic complexity and disambiguating meaning by resolving pronominal reference, performing word sense disambiguation and detecting conventional metaphors (Evans et al., 2014; Orăsan et al., 2018), with some initial efforts towards concept substitutions for images (Barbu et al., 2015). As part of the research project, the tool was evaluated together with end-users with ASD who were shown to find the adapted texts more accessible than the originals. Nevertheless, a major impediment for the automatic evaluation of such systems is the limited amount of userevaluated data. To the best of our knowledge, the only available resources containing a limited amount of such data are the ASD corpus (Yaneva et al., 2016a; Yaneva, 2016), followed by a corpus of easy-to-read documents that were specifically developed for people with cognitive disabilities (Yaneva et al., 2016b) ${ }^{2}$. Constrained by these limitations, some approaches propose to automatically evaluate text simplification systems for people with autism in terms the change in readability of the generated sentences (Evans et al., 2014; Štajner and Saggion, 2013), the incorporation of user-evaluated data into larger corpora (Yaneva et al., 2017), or the use of corpora containing texts for children and language-learners (Štajner et al., 2014). Therefore, very little is known about the perceptions of adults with high-functioning autism on the usefulness of specific simplification strategies.

In the following sections we present a survey on the perceptions of adults with high-functioning autism on the accessibility of user reviews.

\section{Data Collection}

This section presents the way the survey responses were collected.

\footnotetext{
${ }^{1} \mathrm{http}: / /$ www.openbooktool.net/

${ }^{2}$ Note, however, that the latter is not targeted at readers with high-functioning autism
} 


\begin{tabular}{|l|l|}
\hline Question & Possible Answers \\
\hline $\begin{array}{l}\text { 1. Do you read online reviews to determine whether a prod- } \\
\text { uct or service is good or bad? }\end{array}$ & a) Every time b) Very often c) Sometimes d) Rarely e) Never \\
\hline $\begin{array}{l}\text { 2. How many product reviews do you read before coming } \\
\text { to a decision? }\end{array}$ & $\begin{array}{l}\text { a) None b) Less than 10 c) Between 10 and 20 d) More than } \\
\text { 20 e) Most of the available reviews }\end{array}$ \\
\hline 3. In general, do you find understanding product reviews: & a) Very easy b) Easy c) Medium d) Difficult e) Very difficult \\
\hline $\begin{array}{l}\text { 4. In general, how do you find understanding whether the } \\
\text { author approves or disapproves of the described product? }\end{array}$ & a) Very easy b) Easy c) Medium d) Difficult e) difficult \\
\hline $\begin{array}{l}\text { 5. Have you ever felt confused about the meaning of an } \\
\text { online review because the author used irony or sarcasm? }\end{array}$ & a) Very often b) Often c) Sometimes d) Rarely e) Never \\
\hline $\begin{array}{l}\text { 6. Do you think that having a summary of the main points } \\
\text { of all reviews would be: }\end{array}$ & $\begin{array}{l}\text { a) Very helpful b) Helpful c) Moderately helpful d) Not very } \\
\text { helpful e) Not helpful at all }\end{array}$ \\
\hline $\begin{array}{l}\text { 7. Do you feel that there are certain barriers for you with } \\
\text { regards to understanding product reviews? }\end{array}$ & $\begin{array}{l}\text { a) Most of the time b) Often c) Sometimes d) Rarely e) } \\
\text { Never }\end{array}$ \\
\hline $\begin{array}{l}\text { 8. Do you have any recommendations about improving on- } \\
\text { line reviews? Please type your answer in the box below. }\end{array}$ & (open ended question) \\
\hline
\end{tabular}

Table 1: List of the survey questions and their possible answers

\subsection{Survey Structure}

The questions and their possible answers are presented in Table 1. The survey was designed in such a way as to collect information on four main subjects. The first subject was whether or not the participants had any interest in and/or experience with reading online reviews, as well as whether the two groups read a similar amount of surveys before reaching a decision (Q1 and Q2). The second subject was whether or not the participants felt that they experienced any barriers when reading online reviews. This was assessed through two separate questions positioned at different places in the survey. The first question was formulated as a question about rating their experience with understanding the reviews $(\mathrm{Q} 3)$, while the second one directly asked whether they experienced any barriers (Q7). Collecting responses relevant to this subject using two separate types of questions allowed assessing the consistency of the answers between the two. The third subject consisted of structured questions about specific barriers that were both: i) suggested by the literature as potential obstacles for this target population and ii) had corresponding NLP applications developed for these domains (e.g. opinion mining, figurative language identification and text summarization) (Q4, Q5 and Q6). Finally, the last subject was the recommendations that participants had on improving the accessibility of online reviews (Q8), which simultaneously revealed other frustrations that they had which we not accounted for in the structured questions. This was an open-ended question, the responses to which were coded into categories during the analysis stage.

\subsection{Participants}

A total of 57 participants took part in the survey, of whom 24 had a formal clinical diagnosis of autism and 33 were neurotypical control-group participants. All participants from both groups were native speakers of English, with the exception of 1 ASD participant who was native in Romanian and Hungarian but fluent in English. We screened the participants for other conditions affecting reading such as dyslexia and aphasia. None of the controlgroup participants had any of these conditions and 2 of the ASD participants had been diagnosed with dyslexia. The mean age in years of the ASD group was $m=40.08(S D=14.09)$. Number of years spent in formal education were $\mathrm{m}=17.4(\mathrm{SD}=$ 3.26). 18 out of the 24 participants responded to the question "When did you receive your diagnosis?". A total of 7 cases were diagnosed before 2013 following the diagnostic criteria outlined in the DSM-IV. The remaining 11 cases were diagnosed after 2013 following the diagnostic criteria in the DSM-5. The mean age in years of the Control group was $m=40.38(\mathrm{SD}=10.89)$ and number of years spent in formal education were $\mathrm{m}=$ $16.39(\mathrm{SD}=3.11)$.

\subsection{Recruitment Channels}

The majority of the participants with autism were recruited through a UK charity organisation $(\mathrm{N}=$ 17). Another 3 participants were recruited through the Student Enabling Centre at the University of Wolverhampton and the remaining 4 participants were recruited through peer-support groups for people with autism on Facebook. All controlgroup participants were recruited through snowball sampling. 


\subsection{Survey Administration}

All participants completed an online version of the survey. All control-group participants and four ASD-participants were sent a survey link through Survey Monkey ${ }^{3}$. The rest of the ASD participants $(\mathrm{N}=20)$ took part in a larger online reading comprehension experiment and completed the survey as an attachment to that experiment. The questions and their presentation was identical in both platforms.

First, all participants read an information sheet and ticked "Yes" to a question asking for their informed consent to take part in the research. After that the participants were asked for their age in years, number of years spent in formal education and whether or not they had been diagnosed with any of the following: Autism Spectrum Disorder, Dyslexia, Aphasia. If they ticked "Yes" to any of these they were required to state the year in which they had received their formal diagnosis. Another answer options was "No", in which case the participant was assigned to the Control group. The next question assessed whether or not the participant was a native speaker of English. If not, they were required to state their level of fluency in English and their mother tongue. Once information about the demographic characteristics of the participants was collected, they proceeded to answering 7 multiple-choice questions and one open-ended question related to online product reviews.

\section{Results}

This section presents the main results from each question of the survey.

Q1: Do you read online reviews to determine whether a product or service is good or bad? All participants who took part in the survey had experience with reading online reviews for products and services. An equal number of ASD-group participants chose the answer options Sometimes $(33.33 \%, \mathrm{~N}=8)$ and Very often $(33.33 \%, \mathrm{~N}=8)$. Another 7 participants chose the option Every time $(29.17 \%, \mathrm{~N}=7)$ and only 1 participant chose the option Rarely $(4.17 \%, \mathrm{~N}=1)$. More than half of the control-group participants said they read online reviews Very often $(54.55 \%, \mathrm{~N}=18)$ or Every time $(15.15 \%, \mathrm{~N}=5)$. Nine control participants said they read reviews Sometimes $(27.27 \%, \mathrm{~N}=$

\footnotetext{
${ }^{3}$ https://www.surveymonkey.com
}

Figure 1: In general, do you find understanding product reviews:

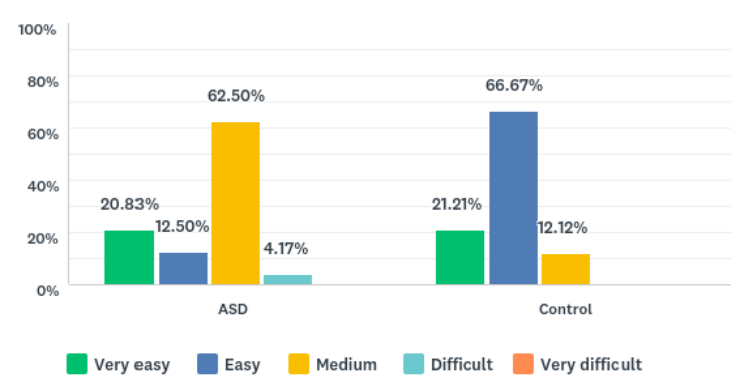

9) and one participant selected the option Rarely $(3.03 \%, \mathrm{~N}=1)$.

Q2: How many product reviews do you read before coming to a decision? The majority of the participants from both groups indicated that they read less than 10 reviews before coming to a decision. The answer distribution for the ASD group was $65.22 \%(\mathrm{~N}=15)$ for the option Less than 10 , $21.74 \%(\mathrm{~N}=5)$ for the option Between 10 and 20 , and $8.7 \%(\mathrm{~N}=2)$ for the option More than 20. One participant had selected the answer Most of the available reviews $(4.35 \%, \mathrm{~N}=1)$. For the Control group, $69.7 \%(\mathrm{~N}=23)$ of the participants chose Less than $10,24.24 \%(\mathrm{~N}=8)$ chose Between 10 and $20,3.03 \%(\mathrm{~N}=1)$ chose Most of the available reviews, and, surprisingly, $3.03 \%(\mathrm{~N}=1)$ chose None. The answer of this last participant contradicts the results from the previous question where no participant chose the option Never.

Q3:In general, do you find understanding product reviews ... There was a statistically significant association between the perceived level of understanding and the group type, where the participants with ASD reported greater difficulty with understanding product reviews $\left(\chi^{2}(3)=21.25, p\right.$ $<0.0001)$. The answer distributions are presented in Figure 1.

Q4: In general, how do you find understanding whether the author approves or disapproves of the described product? Similar to the previous question, there was a statistically significant association between the perceived understanding of the author's opinion and the group type, where the participants with ASD reported greater difficulty with understanding what the opinion was $\left(\chi^{2}(3)=11.94, p=0.008\right)$. The answer distribution for the two groups is presented in Figure 2. 
Figure 2: In general, how do you find understanding whether the author approves or disapproves of the described product?

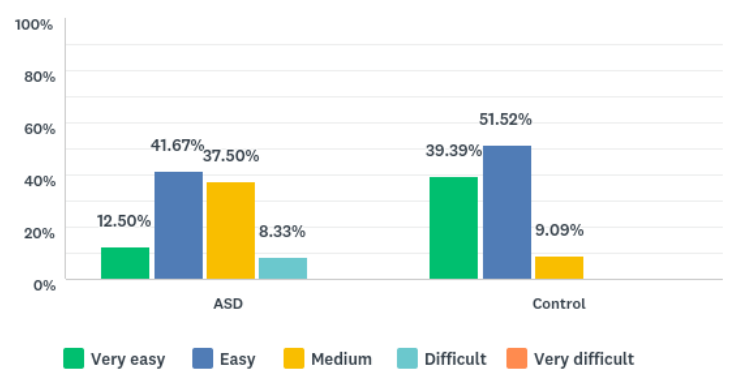

Figure 3: Have you ever felt confused about the meaning of an online review because the author used irony or sarcasm?

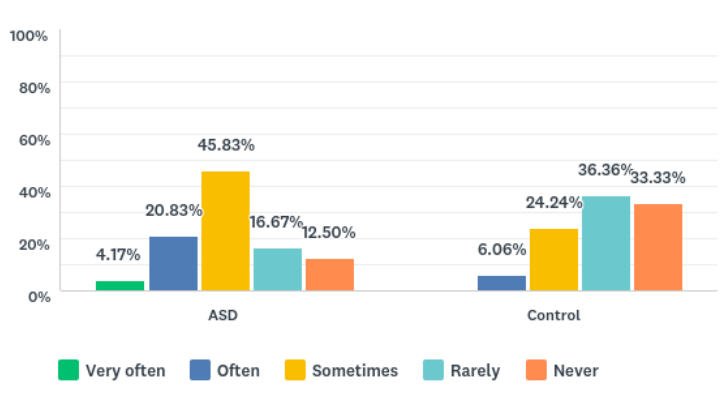

Q5: Have you ever felt confused about the meaning of an online review because the author used irony or sarcasm? The answers to this question were very diverse, however, there was a statistically significant association between the group type and the selected answers $\left(\chi^{2}(4)=\right.$ $10.16, p=0.038)$. While $33.33 \%(\mathrm{~N}=11)$ of the control group said that they had never felt confused by irony or sarcasm in online reviews, this corresponded to only $12.5 \%(\mathrm{~N}=3)$ of the ASD group. The rest of the answer distributions are presented in Figure 3.

Q6: Do you think that having a summary of the main points of all reviews would be ... Large proportions of both groups ${ }^{4}$ reported that they find the idea of summary of the main points of all reviews either Very helpful $(36.36 \%, \mathrm{~N}=8$ of the ASD group and $45.45 \%, \mathrm{~N}=15$ of the Control group) or Helpful $(45.45 \%, \mathrm{~N}=10$ of the ASD group and $39.39 \%, \mathrm{~N}=13$ of the Control group). Moderately helpful and Not very helpful were selected by an equal number of people from both groups, namely $9.09 \%, \mathrm{~N}=2$ of the ASD group

\footnotetext{
${ }^{4}$ Two participants from the ASD group did not give an answer to this question
}

Figure 4: Do you feel that there are certain barriers for you with regards to understanding product reviews?

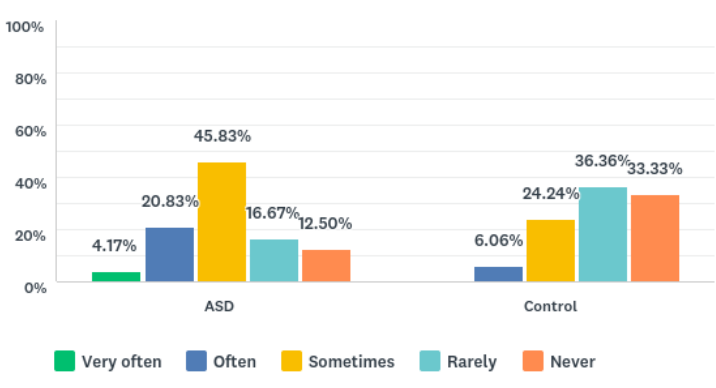

and $6.06 \%, \mathrm{~N}=2$ of the Controls. Finally, one participant from the Control group selected the option Not helpful at all $(3.03 \%, \mathrm{~N}=1)$. There were no significant differences between the opinions of the two groups $\left(\chi^{2}(4)=1.37, p=0.848\right)$.

Q7: Do you feel that there are certain barriers for you with regards to understanding product reviews? Analysis of the responses to this question revealed that the participants with autism significantly more often felt that there are barriers to their comprehension of online reviews ${ }^{5}$ $\left(\chi^{2}(3)=12.92, p=0.005\right)$. The percentages for each answer option are presented in Figure 4.

Q8: Do you have any recommendations about improving online reviews? The answers to this open-ended question were manually coded. The responses of the ASD participants were grouped into the two main categories below.

Category 1: Issues related to language and presentation.

- Text length: Long reviews were identified as the most confusing ones by the participants with autism and they would often give up on them because of information overload, e.g. "I don't like those really long reviews as I can't take it in".

- Organisation: Another demand was for the information to be better organised: "I would rather have numbers and star ratings to support any language."; "Subheadings for what to review; i.e. cost, appearance, functionality etc."; "Bullet points or a summary of the review at the top of the review would be brilliant", and "Having the positive and negatives in a table would be really helpful for me".

\footnotetext{
${ }^{5}$ One person with autism did not give an answer to this question.
} 
Category 2: Issues related to interpretation

- Trustworthiness: Another general issue was anxiety over not being able to decide which reviews are truthful and which ones might be biased or should not be taken seriously. "I would also like to know who the reviewers are and their bias! I find it difficult to trust unknown sources."

- Focus on facts instead of emotion: There was a clear preference for facts to avoid confusion: "Stating facts rather than how it made you feel".

- Exaggeration: Although this was mentioned by only one participant, they explained at length that reviews containing exaggeration and jokes were very confusing for them.

The responses of the control group were mostly related to better organisation and the inclusion of summaries and subheadings.

\section{Discussion}

The results from the survey revealed two important points: i) that the ASD group does perceive reviews as being more challenging to comprehend, confirming the need for adaptation efforts in this domain and ii) the specific aspects in which they find the reviews challenging, together with recommendations for their improvement.

First, participants with and without autism alike had a similar disposition towards reading product reviews and the amount of reviews they read before coming to a decision (less than 10). However, the group with autism consistently perceived online product reviews as being more difficult to understand compared to the control group (Q3). Furthermore, significantly more people from the ASD group felt that there were certain barriers to understanding product reviews compared to the controlgroup participants (Q7), which was a control question that assessed similar information as Q3 (general understanding of product reviews). Formulating this query in two different ways gave consistent results about the perceived difficulty of online reviews for people with autism, which was significantly higher compared to controls.

With regards to specific aspects of the reviews that were potentially challenging, there were significant between-group differences in terms of the understanding of the author's opinion of the product (Q4) and the use of irony and sarcasm (Q5). This suggests that adaptation strategies related to sentiment analysis or opinion mining, together with figurative language identification would be suitable for this domain and target population. Both groups felt strongly in favour of having a summary of the main points of all reviews (Q6), indicating that text summarisation would also be helpful for improving accessibility.

The open-ended question revealed even more aspects that need to be improved, including the length of the text, the lack of consistent structure and the use of exaggeration (Q8). Again, summarisation and topic modeling could help improve the structure of the reviews and potentially present them as a populated table of characteristics that has a consistent structure. An interesting addition was the issue with trusting the reviews. While relevant to all, this may be particularly challenging for individuals on the spectrum due to overall comprehension issues. Therefore, another application that would be particularly helpful for this group of web users would be the detection of fake reviews. While less applicable to a broad type of texts, detecting fake reviews or posts is also relevant to improving the accessibility of social media by making it a safer space.

It is important to note that these results reflect the perceived experiences of the participants rather than their actual processing of product reviews. It is therefore possible that the results from evaluation studies of specific text adaptation strategies may point out to different outcomes. Nevertheless, the perceived experiences of the target group should always be taken into consideration when developing technical solutions and gaining insight into what these are is the first step towards making the web more accessible.

\section{Conclusion}

We conducted a survey with 24 participants with high-functioning autism and 33 neurotypical control participants on their experiences with reading online product reviews. The results showed that both groups were interested in reading reviews before making a purchasing decision but that the ASD group perceived comprehending the reviews to be significantly more challenging. Appropriate strategies for making the reviews more accessible were clarifying the opinion of the author, identify- 
ing any figurative language and summarising the main points of the review, together with enhancing the way the information is structured, as well as flagging reviews that are not trustworthy.

\section{References}

American Psychiatric Association. 2013. Diagnostic and Statistical Manual of Mental Disorders (5th ed.).

Eduard Barbu, M Teresa Martín-Valdivia, Eugenio Martínez-Cámara, and L Alfonso Ureña-López. 2015. Language technologies applied to document simplification for helping autistic people. Expert Systems with Applications, 42(12):5076-5086.

Simon Baron-Cohen. 2000. Theory of mind and autism: A fifteen year review. Understanding other minds: Perspectives from developmental cognitive neuroscience, 2:3-20.

Traolach S Brugha, Sally McManus, John Bankart, Fiona Scott, Susan Purdon, Jane Smith, Paul Bebbington, Rachel Jenkins, and Howard Meltzer. 2011. Epidemiology of autism spectrum disorders in adults in the community in england. Archives of general psychiatry, 68(5):459-465.

Sukru Eraslan, Victoria Yaneva, Yeliz Yesilada, and Simon Harper. 2017. Do web users with autism experience barriers when searching for information within web pages? In Proceedings of the 14th Web for All Conference on The Future of Accessible Work, page 20. ACM.

Sukru Eraslan, Victoria Yaneva, Yeliz Yesilada, and Simon Harper. 2019. Web users with autism: eye tracking evidence for differences. Behaviour \& Information Technology, 38(7):678-700.

Richard Evans, Constantin Orasan, and Iustin Dornescu. 2014. An evaluation of syntactic simplification rules for people with autism. Association for Computational Linguistics.

Rebecca Eynon and Helen Margetts. 2007. Organisational solutions for overcoming barriers to egovernment.

L. Fretwell, J. Stine, H. Sethi, and A. Noronha. 2013. 'catch and keep' digital shoppers how to deliver retail their way. CISCO Internet Business Solutions Group.

Geert Freyhoff, Gerhard Hess, Linda Kerr, Bror Tronbacke, and Kathy Van Der Veken. 1998. Make it simple.

Happe, F., and Frith, U. 2006. he weak coherence account: Detail focused cognitive style in autism spectrum disorder. Journal of Autism and Developmental Disorders, 36:5-25.
Gilbert MacKay and Adrienne Shaw. 2004. A comparative study of figurative language in children with autistic spectrum disorders. Child Language Teaching and Therapy, 20(13).

I.M. O‘Connor and P.D. Klein. 2004. Exploration of Strategies for Facilitating the Reading Comprehension of High-Functioning Students with Autism Spectrum Disorders. Journal of autism and developmental disorders, 34(2).

Constantin Orăsan, Richard Evans, and Ruslan Mitkov. 2018. Intelligent text processing to help readers with autism. In Intelligent Natural Language Processing: Trends and Applications, pages 713-740. Springer.

Sanja Štajner, Richard Evans, and Iustin Dornescu. 2014. Assessing conformance of manually simplified corpora with user requirements: the case of autistic readers. In Proceedings of the Workshop on Automatic Text Simplification-Methods and Applications in the Multilingual Society (ATS-MA 2014), pages 53-63.

Sanja Štajner and Horacio Saggion. 2013. Readability indices for automatic evaluation of text simplification systems: A feasibility study for spanish. In Proceedings of the Sixth International Joint Conference on Natural Language Processing, pages 374-382.

Elisabeth M Whyte, Keith E Nelson, and K Suzanne Scherf. 2014. Idiom, syntax, and advanced theory of mind abilities in children with autism spectrum disorders. Journal of Speech, Language, and Hearing Research, 57(1):120-130.

Victoria Yaneva. 2016. Assessing text and web accessibility for people with autism spectrum disorder. Ph.D. thesis.

Victoria Yaneva, Le Ha, Sukru Eraslan, and Yeliz Yesilada. 2019. Adults with high-functioning autism process web pages with similar accuracy but higher cognitive effort compared to controls.

Victoria Yaneva, Le An Ha, Sukru Eraslan, Yeliz Yesilada, and Ruslan Mitkov. 2018. Detecting autism based on eye-tracking data from web searching tasks. In Proceedings of the Internet of Accessible Things, page 16. ACM.

Victoria Yaneva, Constantin Orasan, Richard Evans, and Omid Rohanian. 2017. Combining multiple corpora for readability assessment for people with cognitive disabilities. In Proceedings of the 12th Workshop on Innovative Use of NLP for Building Educational Applications, pages 121-132.

Victoria Yaneva, Irina Temnikova, and Ruslan Mitkov. 2016a. A corpus of text data and gaze fixations from autistic and non-autistic adults. In Proceedings of the Tenth International Conference on Language Resources and Evaluation (LREC 2016), pages 480487. 
Victoria Yaneva, Irina Temnikova, and Ruslan Mitkov. 2016b. Evaluating the readability of text simplification output for readers with cognitive disabilities. In Proceedings of the Tenth International Conference on Language Resources and Evaluation (LREC 2016), pages 293-299. 